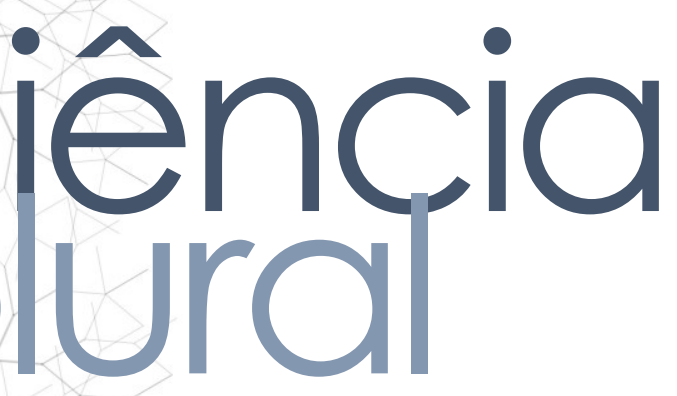

\title{
ASPECTOS CLÍNICO-PATOLÓGICOS DE QUEILITE GRANULOMATOSA: RELATO DE CASO
}

\section{Clinical and pathological aspects of Granulomatosis Queilite: Case Report}

Juliana Campos Pinheiro || Universidade Federal do Rio Grande do Norte | | E-mail: julianapinheiroodonto92@gmail.com

Deborah Lambert Gondim Moreira || Universidade Federal do Rio Grande do Norte || E-mail: deborah.gondim@hotmail.com

Everton Freitas de Morais || Universidade Federal do Rio Grande do Norte | | E-mail: evertonfreitas2@hotmail.com

Gabriel Gomes da Silva || Universidade Federal do Rio Grande do Norte | | E-mail: silvagg94@gmail.com

Jabes Genndedyr da Cruz Lima || Universidade Federal do Rio Grande do Norte || E-mail: jabes.gennedyr@hotmail.com

Carlos Augusto Galvão Barboza || Universidade Federal do Rio Grande do Norte | | E-mail: carlosaugusto2000@hotmail.com

Ana Miryam Costa de Medeiros || Universidade Federal do Rio Grande do Norte || E-mail: anamiryamc.medeiros@gmail.com

Lélia Maria Guedes Queiroz || Universidade Federal do Rio Grande do Norte || E-mail: lmgqueiroz@hotmail.com

Autor responsável pela correspondência

Juliana Campos Pinheiro || E-mail: julianapinheiroodonto92@gmail.com 


\section{RESUMO}

Introdução: A queilite granulomatosa (QG) é uma lesão oral rara, apresentando clinicamente inchaço de aspecto granulomatoso no lábio. Objetivo: O presente estudo, tem como objetivo apresentar um relato de caso de QG, tendo em vista, a dificuldade do diagnóstico e tratamento de condições inflamatórias com etiologias desconhecidas que acometem a cavidade oral. Relato de caso: Paciente do sexo feminino, apresentou aumento de volume no lábio superior exibindo sintomatologia dolorosa, porém, sem relatar associações com alterações sistêmicas. Durante o exame clínico notou-se que o lábio superior apresentava fissuras profundas, observando também edema que se estendia da comissura labial, atravessando a linha média. Levantou-se então, a hipótese diagnóstica de QG. Foi realizada uma biópsia incisional para confirmação do diagnóstico. A análise histopatológica evidenciou, presença de granulomas não caseosos e infiltrado inflamatório linfoplasmocitário perivascular. De acordo com os dados clínicos e análise histopatológica foi dado o diagnóstico inicial de QG. Após o diagnóstico inicial de QG, a paciente foi encaminhada para uma avaliação médica, a fim de avaliar possíveis alterações intestinais que pudessem caracterizar a síndrome de Melkersson-Rosenthal ou Cronh, como também possível reação de hipersensibilidade tardia. Conclusões: $O$ cirurgião-dentista deve estar preparado para diagnosticar aumentos de volume significativos nos lábios, a fim de manejar corretamente o tratamento do paciente, estabelecendo desta forma, prognósticos favoráveis para essas condições.

Palavras-Chave: Queilite; Diagnóstico bucal; Patologia Bucal.

\section{ABSTRACT}

Introduction: Granulomatous cheilitis (HQ) is a rare oral lesion, presenting clinically granulomatous swelling on the lip. Objective: This study aims to present a case report of HG, considering the difficulty of diagnosis and treatment of inflammatory conditions with unknown etiologies that affect the oral cavity. Objective: This study aims to present a case report of HG, considering the difficulty of diagnosis and treatment of inflammatory conditions with unknown etiologies that affect the oral cavity. Case report: A female patient presented increased volume in the upper lip showing painful symptoms, but without reporting associations with systemic alterations. During the clinical examination it was observed that the upper lip presented deep fissures, also observing edema that extended from the labial commissure, crossing the middle line. The diagnostic hypothesis of GQ was then raised. An incisional biopsy was performed to confirm the diagnosis. Histopathological analysis revealed the presence of non-caseous granulomas and perivascular lymphoplasmacytic inflammatory infiltrate. According to the clinical data and histopathological analysis the initial diagnosis of GH was given. After initial diagnosis of $\mathrm{HG}$, the patient was referred for medical evaluation to evaluate possible intestinal changes that could characterize Melkersson-Rosenthal syndrome r Cronh, as well as possible late hypersensitivity reactions. Conclusions: The entist should be prepared to diagnose significant volume increases in the lips in order to correctly manage the patient's treatment, thus establishing favorable ognoses for these conditions.

ywords: Cheilitis; Oral diagnosis; Oral pathology. 


\section{Introdução}

As manifestações bucais podem ser os primeiros sinais de alterações sistêmicas, decorrentes de alguma enfermidade ${ }^{1}$. Lesões orais podem indicar o início ou evolução de alguma doença, portanto, é de fundamental importância o seu diagnóstico precoce ${ }^{2}$. A cavidade oral é o local de manifestação de diversas doenças infecciosas e inflamatórias associadas á doenças sistêmicas, como: líquen plano, lúpus eritematoso, pênfigo e sífilis ${ }^{2,3}$. Além dessas, destacam-se, ainda, as doenças auto-imunes, cujas manifestações bucais são prevalentes².

A queilite granulomatosa $(\mathrm{QG})$ também conhecida como, granulomatose orofacial em lábio foi descrita pela primeira vez por Miescher em 1945, por isso também é chamada de "Miescher's cheilitis", tem um início súbito, curso progressivo, terminando em um aumento crônico dos lábios, sendo umas das principais características dessa lesão $0^{4,5}$.

A QG apresenta-se clinicamente como um inchaço granulomatoso crônico do lábio, com etiologia desconhecida, muitas vezes associada a síndrome de Melkersson-Rosenthal, no qual está associada além da apresentação clínica da QG outros sintomas como paralisia de Bell e fissura lingual ${ }^{4,5}$.

O respectivo trabalho tem como objetivo apresentar um relato de caso, abordando os principais critérios de diagnóstico clínico e histopatológico da QG, levando em consideração, a dificuldade do diagnóstico definitivo e tratamento de condições inflamatórias que acometem a cavidade oral.

\section{Relato de Caso}

Paciente do sexo feminino, 37 anos, feoderma, compareceu a clínica de stomatologia no Departamento de Odontologia da Universidade Federal do Rio Grande do Norte (UFRN), apresentando aumento de volume associado a htomatologia dolorosa em lábio superior direito (Figura 1). 


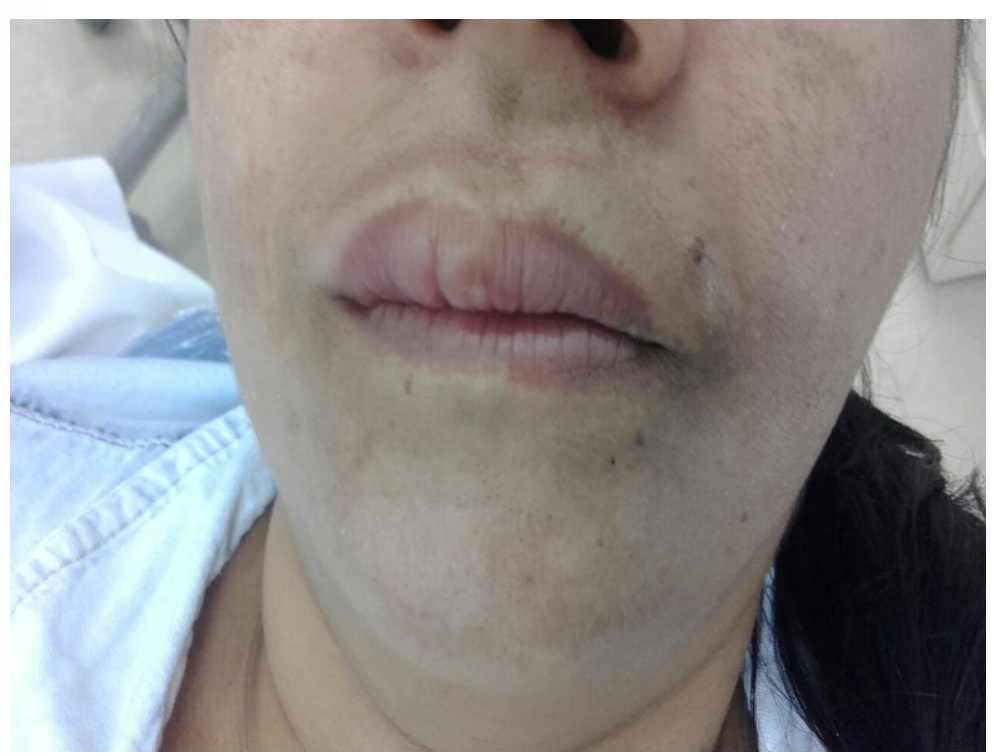

Figura 1 - Aspecto clínico da QG, evidenciando uma lesão edemaciada e com infiltração na região de lábio superior desde a comissura atravessando a linha média. Natal-RN, 2018.

Foi realizada anamnese e exame clínico. De acordo com as informações obtidas na anamnese, a paciente relatou que as alterações bucais começaram aproximadamente há cinco meses, e aumentava de volume gradativamente. A paciente também relatou não possuir alterações sistêmicas e alergias a fatores locais que pudessem estar associadas com as manifestações bucais.

Durante o exame clínico notou-se que o lábio superior apresentava uma temperatura exacerbada e fissuras profundas, observando também edema que se estendia da comissura labial, atravessando a linha média. A lesão apresentava consistência firme e mobilidade ao toque. De acordo com o aspecto clínico e evolução gradativa da lesão, levantou-se a hipótese diagnóstica de QG. Foi realizada uma biópsia incisional para confirmação do diagnóstico através da análise histopatológica.

A análise histopatológica evidenciou, um fragmento de mucosa oral, evestido por epitélio pavimentoso estratificado queratinizado, observando-se lâmina própria a presença de granulomas não caseosos, infiltrado lamatório linfoplasmocitário perivascular e presença de linfócitos 


\section{ciência
purall}

mononucleares dispersos, de forma difusa e em aglomerados. De acordo com os dados clínicos e análise histopatológica foi dado o diagnóstico de QG (Figura 2).
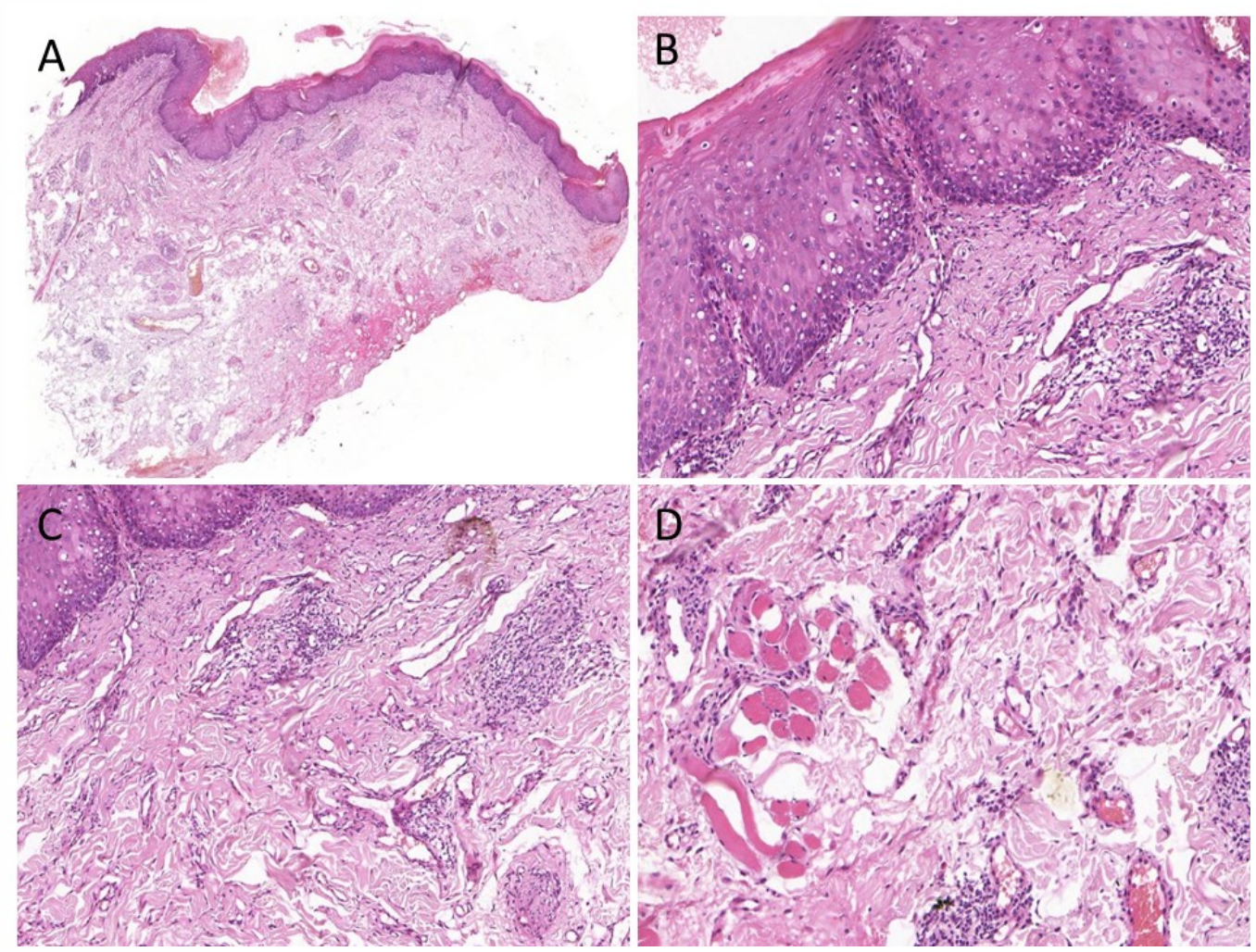

Figura 2 - Aspecto Histopatológico. (A) Fotomicrografia (40x), evidenciando fragmento de mucosa oral, apresentando epitélio pavimentoso queratinizado e com intenso infiltrado inflamatório, assim como vasos sanguíneos congestos, no tecido conjuntivo adjacente. (B) Fotomicrografia (100x), evidenciando espessamento da camada espinhosa e alterações celulares no tecido epitelial. (C) Fotomicrografia (100x), evidenciando rica vascularização e presença de infiltrado inflamatório perivascular, são observados também a presença de linfócitos dispersos, de forma difusa e em grupos. (D) Fotomicrografia (100x), evidenciando camadas mais profundas do tecido conjuntivo, onde se observa a presença de vasos de calibres variados e infiltrado inflamatório predominantemente linfocítico.

Após o diagnóstico inicial de QG, a paciente foi encaminhada para uma avaliação médica: gastroenterologia e alergologista, a fim de avaliar possíveis alterações intestinais que possam caracterizar a síndrome de Melkerssonosenthal ou Cronh, como também algum alérgeno associado relacionado a uma ssível reação de hipersensibilidade tardia. 


\section{Discussão}

Devido à sua extrema raridade e etiologia desconhecida, a QG recebe pouca atenção e pode, muitas vezes, continuar sendo diagnosticada erroneamente $^{6}$. Em 1945, Miescher fez os primeiros estudos relacionados com essa manifestação oral e sistêmica ${ }^{5,6}$. Wiesenfeld em 1985, formulou o termo granulomatose orofacial para classificar as granulomatoses não infecciosas, não necrosantes e distúrbios dos lábios, face e cavidade oral que inclui QG, doença de Crohn e sarcoidose ${ }^{7}$.

A QG se caracteriza como um distúrbio crônico de origem inflamatória, apresentando granulomas não infecciosos e não necróticos, surgindo como um aumento de volume indolor dos lábios, com ou sem edema facial, ocasionando deformidade estética e funcional ${ }^{4,6,8}$. Quando associada com outros achados clínicos como, língua fissurada e paralisia facial, permite-se fazer o diagnóstico da síndrome de Melkersson-Rosenthal. No presente estudo a paciente apresentou sintomas clínicos característicos de QG, incluindo um aumento de volume edemaciado em lábio superior, porém sem comprometimento facial e outros achados que caracterizassem a síndrome de Melkersson-Rosenthal ${ }^{8,9}$.

A tríade completa de acometimentos orais está presente em $8 \%$ a $25 \%$ dos pacientes diagnosticados com síndrome de Melkersson-Rosenthal, embora casos assintomáticos da condição apareça em $47 \%$ dos casos relatados na literatura, sendo que apenas $28 \%$ dos casos, as manifestações de inchaço orofacial ou QG aparecem como única característica da síndrome. Em casos de comprometimento sistêmico o acompanhamento médico é essencial para um melhor prognóstico desta condição ${ }^{10}$.

A etiologia inespecífica da QG é um dos maiores desafios quando se trata esse tipo de lesão oral. Diversas etiologias são citadas na literatura, podendo tar associadas a condições genéticas, inflamatórias, alérgicas e microbianas ${ }^{11}$. Entretanto, existem estudos associando a patogênese da QG com infecções 
odontogênicas ${ }^{10}$. No presente caso, a etiologia da QG era de natureza desconhecida.

Embora a QG possa se desenvolver em qualquer idade, inclusive na infância, ela ocorre mais frequentemente na segunda ou terceira década de vida ${ }^{12,13}$. No estudo de Martínez et al. $^{10}$ a idade dos pacientes variou entre 29 e 74 anos, tais achados da literatura corroboram nosso estudo.

A hipersensibilidade a aditivos alimentares, dentre eles, os compostos de canela, chocolates e benzoatos, foram associados como possíveis agentes etiológicos ${ }^{14,15}$. A sensibilização para metais como ouro e mercúrio também foram correlacionados à patogênese da doença ${ }^{16}$. No caso relatado a paciente foi encaminhada ao alergologista para investigação de uma possível reação de hipersensibilidade tardia.

É de suma importância conhecer o quadro geral do paciente. Tendo em vista que, muitas vezes, as manifestações orais são um reflexo da condição sistêmica ${ }^{1}$, entretanto em nosso caso, a paciente relatou não apresentar patologias de base, sendo encaminhada para avaliação do gastroenterologista.

O inicio das manifestações clínicas podem ter inicio com a formação de edema na região do vermelhão do lábio, podendo se estender em toda região médio-inferior da face ${ }^{5}$. A associação do edema com profundas fissuras no lábio facilita o acúmulo de saliva, ocasionando irritabilidade da mucosa ${ }^{8}$. No presente caso, o lábio superior da paciente apresentou aumento de volume, fissuras labiais profundas, assim como, irritabilidade na mucosa adjacente, corroborando os achados da literatura.

Innocenti et al. ${ }^{6}$ observaram em seu estudo, que os lábios dos pacientes acometidos por QG mantinham os padrões de normalidade, entretanto, em utros pacientes, os lábios apresentavam-se firmes devido a intensa inflamação cal. No presente caso, foi observado a permanência dos padrões de rmalidade na coloração e consistência labial da paciente. 
A QG, geralmente afeta o lábio superior e, menos frequentemente, o lábio inferior. No entanto, a doença também pode afetar outras regiões orais incluindo a mucosa oral, gengivas, língua, faringe e laringe ${ }^{13}$. No presente estudo, a lesão estava localizada apenas em lábio superior, não incluindo outras regiões orais.

As manifestações clínicas da QG vão se agravando gradualmente, podendo apresentar alguns outros sintomas como: descamação, vesículas e/ou pústulas na borda do vermelhão do lábio6. Casos de QG podem evoluir por anos, atingindo condições críticas. No caso relatado a paciente procurou atendimento odontológico antes que se observasse complicações graves,

O diagnóstico de QG se baseia em achados clínicos e histopatológicos. Os achados histopatológicos podem revelar granulomas não necrosantes, edema, linfangiectasia e infiltração linfocitária perivascular. Granulomas não caseosos podem não se apresentar na derme, especialmente nos estágios iniciais de QG, por isso a sua ausência não descarta o seu diagnóstico ${ }^{6}$. No presente caso, foi evidenciado histopatologicamente, a presença de um intenso infiltrado inflamatório, vasos sanguíneos congestos e uma rica vascularização no tecido conjuntivo adjacente.

O diagnóstico diferencial da QG inclui, outras doenças que envolvem a presença de edema facial, condições causadas por granulomas, e também, as formas crônicas de macrocerilia. Vários estudos associam a QG a Doença de Crohn ${ }^{17}$, entretanto esta associação ainda não está completamente elucidada. No presente caso, não foi possível associar a QG com a Doença de Crohn. A paciente foi encaminhada para o gastroenterologista, a fim de avaliar possíveis alterações intestinais que poderiam caracterizar a doença de Cronh. A mesma não retornou para confirmação.

O tratamento da QG apresenta dificuldades para ser realizado. As terapias cais são desempenhadas, levando em consideração a individualidade de cada 05. O tratamento farmacológico pode ser realizado utilizando corticosteróides 
locais e sistêmicos. Os corticóides têm sido amplamente utilizados e, atualmente, têm sido relatado, uma boa resposta tecidual com o uso de injeção intralesional de triancinolona. Álvarez-Garrido et al. ${ }^{17}$ demonstraram em seu estudo, alternativas para diminuir o uso de corticoides, reduzindo os efeitos colaterais do mesmo, apresentando outros tipos de terapias para os casos de QG, utilizando outros medicamentos como: clofazimina, a hidroxicloroquina, a sulfasalazina e a colchicina $^{5}$. Terapias utilizando a a combinação de triancinolona intralesional e clofazimina ou dapsona, tem demonstrado melhores resultados no tratamento da $\mathrm{QG}^{6}$. No presente caso, não foi definido a terapia para o tratamento da QG, uma vez que a paciente foi encaminhada para avaliação médica, afim de elucidar e real etiologia da QG.

Em casos severos de QG, que ocorrem deformidade labial, com ou sem comprometimento da face, os tratamentos farmacológicos podem ser insuficientes. Nessas situações são recomendadas cirurgias plásticas reparadoras ${ }^{5,6}$.

\section{Conclusões}

O cirurgião-dentista deve estar preparado para diagnosticar aumentos de volume significativos nos lábios a fim de manejar corretamente o paciente, estabelecendo o melhor tratamento. Podemos constatar a dificuldade de se estabelecer um tratamento para QG devido aos diversos fatores etiológicos que podem estar associados a lesão. O tratamento da QG deve ser realizado de acordo com as particularidades individuais de cada etiologia, variando de acordo com o grau de desenvolvimento. Os tratamentos podem ser realizados com uso de corticosteróides ou até mesmo cirurgias reparadoras em casos mais graves, no presente estudo, a paciente foi encaminhada para avaliação médica afim de descobrir a real etiologia da QG. O trabalho de uma equipe multiprofissional luindo profissionais da área médica, é de suma importância quando se leva consideração os possíveis comprometimentos sistêmicos que podem estar 
associados com a lesão, estabelecendo desta forma, o tratamento adequado e um melhor prognóstico para essas condições orais.

\section{Referências}

1. Ribeiro BB, Guerra LM, Galhardi WMP, Cortellazzi KL. Importância do reconhecimento das manifestações bucais de doenças e de condições sistêmicas pelos profissionais de saúde com atribuição de diagnóstico. Odonto Jundiaí 2012; 20: 61-70. 2012.

2. Shitara PPL. A percepção dos médicos do conjunto hospitalar de Sorocaba em relação à importância da saúde oral. Universidade Estadual de Campinas 2008.

3. Rai B. Systemic Effect of Oral Disease. The Internet Journal of Family Practice 2006; 5: 1.

4. Braun O, Plewig G, H, Wolff H, Burgdorf WHC. Dermatology 2000. 2nd edition. Heidelberg: Springer-Verlag.

5. Mari CRD, Amaral CB, Wittke EI, Rocha DS, Castilhos M, Bialeski N. Queilite granulomatosa. Saúde 2004; 30: 65-68.

6. Innocenti A, Innocenti M, Taverna M, Melita D, Mori F, Ciancio F, Giorg V, Parronchi P, Vultaggio A, Matuccif A. Miescher's cheilitis: Surgical management and long term outcome of an extremely severe case. Int J Surg Rep 2017:241-244.

7. WIesenfeld D, Ferguson MM, Mitchell DN, Mcdonald DG, Soully C, Cochran K. Orofacial granulomatosis. A clinical and pathological analysis. Q J Med 1985; 54:101-113.

Kumar V. Robbins: Patologia básica. Elsevier Editora Ltda. 9ed. 2013.

Tilakaratne WM, Freysdottir J, Fortune F. Orofacial granulomatosis: view on aetiology and pathogenesis. J Oral Pathol Med 2008; 37:191-195. 
10. Martínez ML, Azada JM, Pérez LJ, López MT, Rodríguez M, Faura C. Granulomatous Cheilitis: A Report of 6 Cases and a Review of the Literature. Actas Dermosifiliogr 2012; 103:718-724.

11. Gharbi A, Sonthaklia S. Cheilitis Granulomatosa (Miescher Melkersson Rosenthal Syndrome). StatPearls Publishing 2018.

12. Dodi I, Verri R, Brevi B, Bonetti L, Balestrier A, Saracino A. A monosymptomatic Melkersson-Rosenthal syndrome in an 8- year old boy. Acta Biomed 2006; 77: 20-23.

13. Vanderwaal RI, Schulten EA, Vandescheur MR, Wauters IM, Starink TM, Van derwaal I. Cheilitis granulomatosa. J Eur Acad Dermatol Venereol 2001; 15: 519-523.

14. Taibjee S, Prais L, Foulds I. Orofacial granulomatosis worsened by chocolate: results of patch testing to ingredients of Cadbury's chocolate. Br J Dermatol 2004; 150: 595.

15. Fitzpatrick L, Healy CM, Mccartan BE, Flint SR, Mccreary CE, Rogers S. Patch testing for food-associated allergies in orofacial granulomatosis. J. Oral Pathol Med 2011; 40: 10-13.

16. Lazarov A, Kidron D, Tulchinsky Z, Minkow B. Contact orofacial granulomatosis caused by delayed hypersensitivity to gold and mercury. J Am Acad Dermatol 2003; 49: 1117-1120.

17. Álvarez H, Pericet L, Martínez G, Tejerina JA, Peral I, Miranda A. Crohn's disease and cheilitis granulomatosa: Role of silicone fillers. J Am Acad Dermatol $2011 ; 65 ; 239-241$.

bmetido: 10/09/2018

provado: 30/04/2019 